\title{
India Losing Battle Against Multi Drug Resistant Tuberculosis
}

\author{
Anjali Saxena $\cdot$ Udita Mukherjee $\cdot$ Ankita Dua \\ Rup Lal
}

(C) Association of Microbiologists of India 2012

Tuberculosis (TB), an infectious air borne disease, is a major health problem worldwide. It is the second most lethal disease, next only to AIDS, globally [1]. Besides this, another growing concern is the development of the Multi Drug Resistant (MDR) forms of tuberculosis which have developed due to partial or incomplete treatment. This form of tuberculosis is resistant to the most important primary drugs; isoniazid and rifampicin.

As a result the mortality rates due to MDR-TB appear to increase in leaps and bounds, and this ominous phenomena is generally seen in developing and under developed parts of the world. MDR-TB results either from primary infection or may develop in the course of a patient's treatment. TB, however, is a curable disease but MDR-TB requires much stronger treatment for up to 2 years, with second line treatment, for which the drugs are limited and are not always available [2]. MDR-TB is highly prevalent in people infected with HIV [3], as a suppressed immune system makes HIV patients more vulnerable to drug resistant TB.

India is reported to be highly burdened with TB cases, accounting for $21 \%$ of the global incidence, with an estimated 2 million cases, annually. It is also estimated by the World Health Organization that 320,000 people die from TB each year in India. In January 2012, India became the second country in the world to report a strain of TB that is resistant to all known drugs, a form termed as the Totally Drug Resistant TB (TDR-TB). Despite the loss of millions of lives, TB remains one of the most neglected diseases due to lack of proper treatment. Especially, there is no commercially available drug available to treat MDR-TB yet. In a recent shocking

\footnotetext{
A. Saxena $\cdot$ U. Mukherjee $\cdot$ A. Dua $\cdot$ R. Lal $(\bowtie)$

Department of Zoology, University of Delhi, Delhi 110 007, India

e-mail: ruplal@gmail.com
}

disclosure by WHO, India is home to around 73,000 MDR-TB patients, supposedly the highest numbers in the world. However, only 1,660 cases were notified and 1,136 cases are being treated. The statistics are clear indicators of inappropriate treatment and incoherence of the patients to the treatment regime, which results from lack of awareness.

For improving the status of TB, in May 2012, India took a step forward and declared TB to a notifiable disease, where all doctors, caretakers treating a TB patient must report every TB case to the government. In September 2012, guidelines were set on how TB cases are supposed to be notified.

Inadequate care and mismanagement are primary reasons for the emergence of this deadly disease. Despite launching new programs, India's Revised National Tuberculosis Control Program (RNTCP), by the Indian government, addressing the issues of TB along with its resistant forms, default rates in the MDR-TB program remains high. This scenario will continue to spread drug resistant TB among communities and will further amplify resistance.

Looking at the current scenario, there is a compelling and urgent need for novel TB drug regimens, effective and safe vaccines to keep the disease under control. Further, launching a more effective TB control programme in India will be a steping stone towards controlling the disease which is becoming deadlier by the day.

\section{References}

1. World Health Organization (2010) Fact Sheet No.104: Tuberculosis. Geneva, WHO http://www.who.int/mediacentre/factsheets/ fs 104/en/print.html

2. Crofton J, Chaulet P, Maher D (1997) Guidelines for the management of drug-resistant Tuberculosis. Geneva, WHO, pp 31-37

3. Demissie M, Lemma E, Gebeyehu M, Lindtjorn B (2001) Sensitivity to anti-tuberculosis drugs in HIV-positive and -negative patients in Addis Ababa. Scand J Infect Dis 33:914-919 